\title{
Microsporidian Infection in Mosquitoes (Culicidae) Is Associated with Gut Microbiome Composition and Predicted Gut Microbiome Functional Content
}

\author{
Artur Trzebny $^{1}$ [ $\cdot$ Anna Slodkowicz-Kowalska² $\cdot$ Johanna Björkroth ${ }^{3} \cdot$ Miroslawa Dabert $^{1}$
}

Received: 10 April 2021 / Accepted: 10 December 2021 / Published online: 23 December 2021

(c) The Author(s) 2021

\begin{abstract}
The animal gut microbiota consist of many different microorganisms, mainly bacteria, but archaea, fungi, protozoans, and viruses may also be present. This complex and dynamic community of microorganisms may change during parasitic infection. In the present study, we investigated the effect of the presence of microsporidians on the composition of the mosquito gut microbiota and linked some microbiome taxa and functionalities to infections caused by these parasites. We characterised bacterial communities of 188 mosquito females, of which 108 were positive for microsporidian DNA. To assess how bacterial communities change during microsporidian infection, microbiome structures were identified using 16S rRNA microbial profiling. In total, we identified 46 families and four higher taxa, of which Comamonadaceae, Enterobacteriaceae, Flavobacteriaceae and Pseudomonadaceae were the most abundant mosquito-associated bacterial families. Our data suggest that the mosquito gut microbial composition varies among host species. In addition, we found a correlation between the microbiome composition and the presence of microsporidians. The prediction of metagenome functional content from the 16S rRNA gene sequencing suggests that microsporidian infection is characterised by some bacterial species capable of specific metabolic functions, especially the biosynthesis of ansamycins and vancomycin antibiotics and the pentose phosphate pathway. Moreover, we detected a positive correlation between the presence of microsporidian DNA and bacteria belonging to Spiroplasmataceae and Leuconostocaceae, each represented by a single species, Spiroplasma sp. PL03 and Weissella cf. viridescens, respectively. Additionally, W. cf. viridescens was observed only in microsporidian-infected mosquitoes. More extensive research, including intensive and varied host sampling, as well as determination of metabolic activities based on quantitative methods, should be carried out to confirm our results.
\end{abstract}

Keywords Indicator taxon analysis $\cdot$ Metagenome functional content $\cdot$ Microsporidia $\cdot$ Mosquito gut microbiota $\cdot$ Microsporidian infection · PICRUSt2 $\cdot$ Spiroplasma $\cdot$ Weissella

\section{Introduction}

Microsporidians (Microsporidia) are among the most widespread, obligate intracellular parasites, causing mainly zoonotic or waterborne infections in humans, but able to

Artur Trzebny

arturtrzebny@amu.edu.pl

1 Molecular Biology Techniques Laboratory, Faculty of Biology, Adam Mickiewicz University, Poznan, Poland

2 Department of Biology and Medical Parasitology, Poznan University of Medical Sciences, Poznan, Poland

3 Department of Food Hygiene and Environmental Health, Faculty of Veterinary Medicine, University of Helsinki, Helsinki, Finland infect almost all animal phyla [1]. Among 1700 described microsporidian species [2], more than 700 were isolated from insects [3], with mosquitoes (Culicidae) the most common $[4,5]$. It is noted that microsporidians represent one of the largest and most diverse groups of parasites associated with mosquito populations in nature [4].

Mosquitoes can act as vectors for many disease-causing viruses and parasites and even carry and transmit multiple pathogens in a single host, creating numerous opportunities for interactions among vertebrate hosts, vectors, and pathogenic organisms. These interactions can occur on multiple levels and may ultimately affect transmission patterns and disease pathogenesis. One example is the possible immunosuppressive effect of filarial nematodes on malaria leading to reduced Plasmodium infectivity in mosquitoes [6]. The lowering of the efficiency of the infection of viral 
diseases, such as dengue or Zika, was also recorded in the co-occurrence of viruses and entomopathogenic fungi [7] or the bacterial endosymbiont Wolbachia spp. [8]. Moreover, there are reports that the insect microbiome can modulate the vector competence for arboviruses in Aedes and Culex mosquitoes [9].

The effects of microsporidians on the development of disease-causing organisms in mosquitoes have been studied mainly for malaria parasites for developing novel strategies to control mosquito populations or their capability to transmit Plasmodium parasites [10-12]. It has been shown that Vavraia culicis impairs the development of Plasmodium berghei by priming the immune melanisation response in the adult mosquito [11]. However, in mosquitoes, like in other insects, microsporidians also may affect the functions [13] and behaviour of the host, such as decreased lifespan, oviposition [14] or flight activity [15]. Interestingly, some of the mechanisms underlying these changes involve alterations in commensal bacterial species in the host gut microbiome [16, 17]. A recent study showed that infection of Anopheles arabiensis with a vertically transmitted microsporidian species prevented $P$. falciparum transmission because it reduced the establishment of Plasmodium oocysts in the Anopheles midgut and impaired the colonisation of Anopheles salivary glands by Plasmodium sporozoites [10]. Moreover, it has been shown that Nosema ceranae is to some extent associated with changes in gut microbiome structure and its presence is strongly correlated with some gut microbiome members (e.g. Gilliamella spp. in the honey bee, Apis mellifera) [18].

It has been increasingly recognised that microorganisms may participate in host-parasite interactions [19]. The gut microbiome may also have important direct and indirect effects on parasite establishment, including insect hosts [20, 21]. Recently, a proof-of-concept study showed significant changes in the gut bacteriome and mycobiome of the grain beetle (Tenebrio molitor) in association with tapeworm (Hymenolepis diminuta) infection [22]. Like that of other insects, the gut microbiome of mosquitoes mediates the interactions between the host and intestinal parasites by stimulating the host immune responses [23,24] or protecting the host by inhibiting parasites, for example [25, 26]. These interactions have a promising application to prevent pathogen transmission because they can affect the symbiont populations and shape the microbial community structure of the host [27, 28].

Many studies have shown that sex, stage of development, host environment, diet type and pathogenic infection can influence the mosquito microbiota [26, 29-37]. Mosquito larvae live in water and so acquire the majority of their gut microbiota from their environment. Therefore, high water temperature, $\mathrm{pH}$ and oxygen content, and an abundance of residual antibiotics in breeding sites of mosquito larvae shape their microbiome [30-33]. During metamorphosis, the larval midgut bacteria are largely eliminated because of meconium egestion by the newly hatched adult mosquito [26, 29, 34]. Therefore, in adult mosquitoes, the most important factors determining microbiota are the source of blood meal, blood digestion and nectar assimilation or variations in mosquito sex and size [35-37].

Mosquito-microbiome interactions play an important role in mosquito biology, including the development of pathogens $[38,39]$. Symbiotic bacteria affect the development of pathogens via the production of metabolites or by stimulating the host immunological responses [38, 40]. Additionally, it has been shown that the pathogenic bacterium Serratia marcescens as a microbiome member may enhance dengue virus infection [41] or inhibit $P$. berghei infection [28]. It was also demonstrated that Wolbachia spp. may repress [42] or inhibit P. falciparum [43] in Anopheles gambiae. Although mosquitoes are common hosts of microsporidia, there is a lack of data on their effects on the mosquito microbiota. Therefore, the present study aimed to detect changes in mosquito gut microbiota associated with microsporidian infection. As a model, we used mosquitoes collected from natural populations.

\section{Methods}

\section{Material}

For this study, we used 188 DNA isolates extracted from 188 female mosquitoes collected for a previous study [44] between July and August 2016 from the periphery of mixed birch-oak and riparian forests near the city of Poznan, western Poland. Here, we used representatives of five species: Aedes vexans (19), Coquillettidia richiardii (16), Ochlerotatus annulipes (63), O. cantans (77) and O. sticticus (13). Among them, 108/188 (58\%) were positive for one or more microsporidian species (Table 1). Infection with Microsporidium sp. PL01 predominated in all tested mosquito species. Negative control samples from blank extractions, including solution used to wash mosquitoes before DNA extraction, were analysed in the same way as the test samples.

\section{Library Construction and NGS Sequencing}

For 16S rRNA microbial profiling, we used V4F (CGATCA GCAGCCGCGGTAATA) and V4R (ATGGACTACCAG GGTATCTAA) primers targeting the V4 region in prokaryotic 16S rRNA gene [45]. Primers were tailed at 5'-ends with dual-indexed Ion Torrent adapters for sequencing using the Ion Torrent system (Life Technologies, USA). PCR reactions were done in two technical replications, each in a total volume of $10 \mu$, containing Hot FIREPol DNA Polymerase (Solis BioDyne, Estonia), $0.25 \mu \mathrm{M}$ of each primer and $1 \mu \mathrm{l}$ of template DNA. The PCR program was as follows: $95^{\circ} \mathrm{C}$ 
Table 1 DNA isolates from microsporidian-infected and non-infected mosquitoes used in this study. Higher numbers of infected mosquitoes result from co-infection events with more than one microsporidian species

\begin{tabular}{|c|c|c|c|c|c|c|}
\hline \multirow[t]{2}{*}{$\begin{array}{l}\text { Microsporidian } \\
\text { species }\end{array}$} & \multicolumn{6}{|c|}{$\begin{array}{l}\text { Mosquito species } \\
\text { (infection/individuals) }\end{array}$} \\
\hline & $\begin{array}{l}\text { Ae. vexans } \\
(13 / 19)\end{array}$ & $\begin{array}{l}\text { C. richiardii } \\
(9 / 16)\end{array}$ & $\begin{array}{l}\text { O. annulipes } \\
(34 / 63)\end{array}$ & $\begin{array}{l}\text { O. cantans } \\
(44 / 77)\end{array}$ & $\begin{array}{l}\text { O. sticticus } \\
(8 / 13)\end{array}$ & $\begin{array}{l}\text { All mos- } \\
\text { quitoes } \\
(108 / 188)\end{array}$ \\
\hline Amblyospora salinaria & & & & & 1 & 1 \\
\hline Amblyospora sp. & & & 1 & 1 & 1 & 3 \\
\hline Encephalitozoon hellem & & & & 2 & & 2 \\
\hline Enterocytospora artemiae & & & 1 & & & 1 \\
\hline Microsporidium sp. nov. PL01 & 10 & 9 & 31 & 27 & 7 & 84 \\
\hline Nosema adaliae & & & 1 & & & 1 \\
\hline N. ceranae & & & & 2 & & 2 \\
\hline N. chrysorrhoeae/portugal & 2 & 1 & 4 & 16 & & 23 \\
\hline N. pieriae & & 1 & 2 & & & 3 \\
\hline N. thomsoni & 1 & & & & & 1 \\
\hline Nosema sp. CHW-2007a & 2 & 1 & 2 & 1 & 1 & 7 \\
\hline
\end{tabular}

for $12 \mathrm{~min}$, followed by 30 cycles at $95^{\circ} \mathrm{C}$ for $15 \mathrm{~s}, 50{ }^{\circ} \mathrm{C}$ for $1 \mathrm{~min}$ and $72{ }^{\circ} \mathrm{C}$ for $45 \mathrm{~s}$, with a final extension step at $72{ }^{\circ} \mathrm{C}$ for 5 min. After PCR, technical replications were pooled and, for each sample, $3 \mu \mathrm{l}$ was separated by electrophoresis on a $2 \%$ agarose gel to check amplification efficiency. Then, all samples were pooled in equal quantities and then purified using the $2 \%$ E-Gel SizeSelect II Agarose Gels system (Invitrogen, USA), according to the manufacturer's instructions.

DNA concentration and fragment length distribution of the library were established using the High Sensitivity D1000 Screen Tape assay on a 2200 Tape Station system (Agilent, USA). Clonal template amplifications were performed using the Ion Torrent One Touch System II and the Ion Torrent OT2 Kit (Life Technologies) according to the manufacturer's instructions. Sequencing was carried out using the Ion 540 Kit-OT2 and Ion Torrent S5 system on the Ion 540 chip (Life Technologies) according to the manufacturer's instructions.

\section{Read Processing and Data Analysis}

Raw sequence data were pre-filtered using the Ion Torrent Suite software version 5.10.1 (Life Technologies) to remove polyclonal and low-quality sequences. Further bioinformatic analyses were conducted using fastq data and custom workflow. Sequence reads shorter than $200 \mathrm{bp}$ were removed from the dataset using Geneious R11.1.5 (Biomatters Ltd.). Leading and trailing low-quality bases were removed using Trimmomatic version 0.39 [46]. FASTX-Toolkit [47] was used to extract sequences with a minimum of $50 \%$ bases with a quality score of $\geq 25$. Quality-filtered sequences were separated by barcodes and trimmed at 5'- and 3'-ends to exclude PCR primers in Geneious R11.1.5. The singletons ( $<10$ reads) were removed using the FASTX_UNIQUES and SORTBYSIZE algorithms [48]. Chimeras were removed using the default settings in UCHIME2 version 4.2.40 [49].

Operational taxonomic unit (OTU) clustering at $97 \%$ similarity was done in USEARCH version 11.0.667 [48]. Sequences were denoised into zero-radius operational taxonomic units (ZOTUs), and subsequently, a ZOTU table was constructed according to the DENOISING STEPS [49]. The ZOTU table was then corrected for the $16 \mathrm{~S}$ copy number based on the UNBIAS algorithm. Phylogenetic affiliations were analysed by the USEARCH SINTAX algorithm using a confidence threshold of 0.8 [50-53]. ZOTUs were compared against the SILVA database for ARB for small subunit ribosomal RNAs version 138 [54-56]. ZOTUs detected in control samples were used to identify cross-talk errors among the analysed mosquito samples. The UNCROSS2 algorithm was used to remove ZOTUs detected in control samples from the dataset [57]. Then, the reads were normalised by the OTUTAB_RARE algorithm [51] to compare sample diversities.

The functional potential of prokaryotic communities in all samples was predicted using the Phylogenetic Investigation of Communities by Reconstruction of Unobserved States (PICRUSt2) version 2.4.1 software package [58]. The ZOTU table normalised by $16 \mathrm{~S}$ rRNA gene copy number was used for metagenome functional prediction, generating a table of Kyoto Encyclopedia of Genes and Genomes (KEGG) Orthologs (KOs) [59-61]. The predictions were categorised at KEGG Orthology level 1,2 and 3 within the pathway hierarchy of KEGG. As an indicator for the PICRUSt2 prediction accuracy, the Nearest Sequenced Taxon Index (NSTI) for each sample was estimated and calculated 
[62]. The comparison of potential functions among different sample categories was supplemented by the Statistical Analysis of Metagenomic Profiles (STAMP) version 2.1.3 [63].

\section{Amplification of Complete 16S rRNA Gene from Weissella}

To confirm the taxonomic position of the Weissella identified by NGS, a near-complete Weissella $16 \mathrm{~S}$ rRNA gene was amplified using genus-specific S-G-Wei-0121-a-S-20 (CGT GGGAAACCTACCTCTTA) and S-G-Wei-0823-a-A-18 (CCCTCAAACATCTAGCAC) primers [64]. PCR reactions were prepared in two technical replicates, each in a total volume of $10 \mu \mathrm{l}$, containing Hot FIREPol DNA Polymerase, $0.25 \mu \mathrm{M}$ of each primer and $1 \mu \mathrm{l}$ of template DNA. The amplification program was as follows: $95^{\circ} \mathrm{C}$ for $12 \mathrm{~min}$, followed by 35 cycles at $95^{\circ} \mathrm{C}$ for $15 \mathrm{~s}, 60^{\circ} \mathrm{C}$ for $1 \mathrm{~min}$ and $72{ }^{\circ} \mathrm{C}$ for $1 \mathrm{~min}$, with a final extension step at $72{ }^{\circ} \mathrm{C}$ for $10 \mathrm{~min}$. After amplification, technical replications were pooled, and $5 \mu \mathrm{l}$ was analysed by electrophoresis on $1.5 \%$ agarose gel.

Samples containing visible bands were purified with Escherichia coli exonuclease I and FastAP Alkaline Phosphatase (Thermo Scientific, USA) and sequenced using the BigDye v3.1 Kit and ABI Prism 3130XL Genetic Analyzer (Applied Biosystems, USA), following the manufacturer's instructions. Sequence chromatograms were checked for accuracy in Geneious R11.1.5. Then, sequences were compared to GenBank using blastn [65] optimized for highly similar sequences (megablast algorithm) [66].

\section{Spiroplasma Identification Using Phylogenetic Analysis}

To identify the Spiroplasma species isolated from mosquitoes, all 16S sequences assigned to the genus Spiroplasma published in GenBank (www.ncbi.nlm.nih.gov) were used. As close outgroups, the $16 \mathrm{~S}$ sequences of Clostridium ramosum and five Mycoplasma spp. were used. The bacterial strains and GenBank accession numbers for DNA sequences used in the Spiroplasma spp. phylogenetic analysis are provided in Supplementary Table 1.

Sequences were aligned using the L-INS-I algorithm in MAFFT version 7.388 [67], as implemented in Geneious R11.1.5. The best-fit model of DNA evolution $(G T R+I+G)$ was chosen by PartitionFinder2 [68]. Phylogenetic trees were reconstructed using maximum likelihood (ML) in Garli version 2.0 [69] and Bayesian inference (BI) in MrBayes version 3.2.6 [70]. Each BI run of four independent chains was performed in $4 \times 10,000,000$ generations, and the trees were sampled every 1000 generations. The final consensus tree was generated after discarding the burn-in fraction of $0.25 \%$ of initial trees; the average standard deviation of split frequencies dropped below 0.002. Bootstrap support for the ML tree was calculated by using 1000 data replicates as implemented in Garli. The trees were edited in FigTree version 1.4.4 [71] and further edited in Corel Draw X4.

\section{Statistical Analyses}

The Chao1 index and Shannon diversity in individual samples were calculated using the ALPHA_DIV algorithm [51]. Indexes were analysed using one-way ANOVA. The remaining statistical analyses were performed using the $\mathrm{R}$ software version 4.0.2 [72]. Visualization of the heatmap and cluster tree based on unweighted pair-group method with arithmetic median (UPGMA) were conducted in STAMP version 2.1.3 [63]. Statistical analyses were performed using permutation tests implemented in the coin package version 1.4-1 [73]. Comparisons between two independent groups were conducted using the Wilcoxon-Mann-Whitney (WMW) test. Comparisons of more than two groups were conducted using the Kruskal-Wallis test. To assess the difference between two independent groups, a statistic $r$ was used, defined as $r=|Z| / \sqrt{ } \mathrm{N}$, where " $Z$ ' is the WMW test statistic and " $\mathrm{N}$ " is the number of observations. The relationship between the two variables was tested using the Spearman correlation coefficient (rho, $\rho$ ).

The correlation between microbiota composition and mosquito species, in addition to the effects of microsporidian infection and their interaction with mosquito microbiome composition, was tested using the analysis of similarities (ANOSIM) with 999 permutations [74]. Variation in microbial community composition and pathways' structure differentiation was visualised using principal coordinate analysis (PCoA). An indicator species analysis was performed to determine whether taxa were exclusively found in infected or non-infected groups and if these taxa were commonly found in certain treatment groups, as revealed by the A and B components of the indicator species analysis, respectively. The indicator species analysis was performed using the multipatt function within the indicspecies package [75].

Comparisons of the two groups and correlation analysis were tested considering mosquitoes belonging to different species using stratified permutation tests. Additionally, statistical significances among Weissella and mosquito species were calculated based on the chi-square test. Any $p$-values of $\leq 0.05$ were considered significant.

\section{Results \\ Bacterial Communities in Field-Collected Mosquitoes}

After quality-filtering the samples, 18,006,087 reads were yielded. The average read number per sample was 95,777 $(\mathrm{SD}=9$ 343). The clustering of ZOTUs across samples produced 1437 unique ZOTUs. The median of ZOTUs 
was 96 and ranged between 84 and 112 . There were no differences in species richness (Chao1 index; $\mathrm{F}=1.67$; $p>0.848$; Supplementary Fig. 1) and diversity (Shannon diversity; $\mathrm{F}=1.77 ; p>0.758$; Supplementary Fig. 2) between microsporidia-positive and non-infected mosquitoes belonging to different species.

ZOTUs were clustered into 46 families and four higher taxa (Fig. 1). Proteobacteria (59\%; $\mathrm{SD}=12$ ), Bacteroidetes $(19 \% ; \mathrm{SD}=11)$ and Firmicutes $(17 \% ; \mathrm{SD}=8)$ were the most highly abundant phyla associated with mosquitoes tested in this study (Supplementary Fig. 3, Supplementary Table 2). Among them, for both infected and non-infected mosquitoes, the most dominant families were Enterobacteriaceae (infected 19\%, SD =10; non-infected 20\%; $\mathrm{SD}=11$ ), Flavobacteriaceae (infected $17 \%, \mathrm{SD}=11$; noninfected $18 \% ; \mathrm{SD}=11$ ) and Comamonadaceae (infected $8 \%, \mathrm{SD}=6$; non-infected $10 \%$; $\mathrm{SD}=6$ ). Diversity analysis of mosquito microbiota based on the cluster tree using UPGMA showed that the diversity of bacterial communities was determined by mosquito species rather than microsporidian infection (Fig. 1).

Unlike the PCoA results of all mosquito microbiota as a pooled sample (i.e. without separation by host species), which did not group the bacterial communities according to microsporidian infection (Fig. 2A), the ANOSIM significantly differentiated them as associated with this infection $(R=0.09, p<0.001)$ and mosquito species $(R=0.43$, $p<0.001)$. By contrast, the results of PCoA for separate host species showed that the occurrence of microsporidians clearly differentiated the observed microbiome compositions (Fig. 2B-F).

Indicator species analysis for infected and non-infected mosquitoes (Table 2) showed that among the non-infected individuals, the families Aurantimonadaceae, Conexibacteraceae, Cytophagaceae, Dermabacteraceae and Hydrogenophilaceae displayed high values for the exclusive taxon component (A > 0.9). Additionally, the Actinomycetaceae, Halomonadaceae, Methylobacteriaceae, Moraxellaceae, Mycobacteriaceae and Sphingomonadaceae were noticed in all mosquitoes without microsporidians $(\mathrm{B}=1)$. Mosquitoes infected with microsporidians were characterised by the exclusive occurrence of the Leuconostocaceae $(\mathrm{A}=1)$. Moreover, the Acetobacteraceae, Bradyrhizobiaceae, Pseudomonadaceae and Rhizobiaceae were noticed in all infected mosquitoes $(\mathrm{B}=1)$, while the Spiroplasmataceae was associated with the presence of microsporidians due to a high $\mathrm{B}$-component value $(\mathrm{B}>0.9)$.

\section{Gut Microbiome Members Associated with Microsporidian Infection}

Among the Spiroplasmataceae, only one ZOTU, Spiroplasma sp. PL03, was clustered. The sequence represented a
Spiroplasma species that does not have a reference sequence published in databases. Phylogenetic analysis grouped this sequence with other endosymbiotic Spiroplasma spp. (Fig. 3). The prevalence of this species was $100 \%$ in infected and uninfected mosquitoes except for $C$. richiardii in which Spiroplasma was detected only in one infected individual (1.11\%) (Fig. 1, Supplementary Table 3) and its presence in microsporidian-positive mosquitoes was 2.47 to $23.79 \%$ higher than in non-infected ones (Fig. 1, Supplementary Table 4). This result had statistical significance in Ae. vexans, O. cantans and O. sticticus (Supplementary Table 5). However, we did not find a significant correlation between increasing numbers of microsporidian and Spiroplasma sp. sequence reads $(0.1 \leq r \leq 0.27, p=0.216)$ (Supplementary Table 6).

Based on the $16 \mathrm{~S}$ microbial profiling, the only representative ZOTU among Leuconostocaceae was assigned to the genus Weissella, while the almost-complete 16S rRNA gene (GenBank acc. nos. MW892051-MW892067) allowed species identification using BLASTn search, revealing the highest similarity to Weissella viridescens (100\% identities). This bacterium was detected only in microsporidian-positive mosquitoes (Supplementary Fig. 4, Supplementary Table 4). There were no significant differences in $W$. cf. viridescens abundance among mosquito species $\left(\chi^{2}=4.06, p=0.4\right)$, but a strong positive correlation $(0.45 \leq r \leq 0.81, p<0.001)$ between microsporidian and $W$. cf. viridescens frequency was observed (Supplementary Table 6). Furthermore, an increased presence of Spiroplasma sp. PL03 was observed in $O$. annulipes individuals in which microsporidians cooccurred with $W$. cf. viridescens $(Z=-2.51, p=0.007)$ (Supplementary Table 7).

\section{Functional Analysis of Mosquito Microbiota During Microsporidian Infection}

In total, based on the $16 \mathrm{~S}$ amplicon dataset, PICRUSt2 generated 183 functional pathways using KEGG pathway metadata. The PICRUSt 2 metagenome predictions had NSTI scores ranging from 0.001 to 0.182 . The mean NSTI value was 0.049 for infected mosquitoes and 0.044 for noninfected ones (Supplementary Table 8).

Considering the total genes, almost $80 \%$ of them were related to the metabolic pathways and showed significant differences in abundance (Welch's $t$-test, two-sided, $p=0.029$ ) between microsporidian-positive and non-infected mosquitoes. For both infected and non-infected mosquitoes specifically, carbohydrate metabolism, metabolism of terpenoids and polyketides, and amino acid metabolism were the most abundant pathways (>10\%) at level 2 (Fig. 4, Supplementary Table 9).

Principal coordinate analysis based on microbiome predicted functions revealed three groups of mosquitoes 

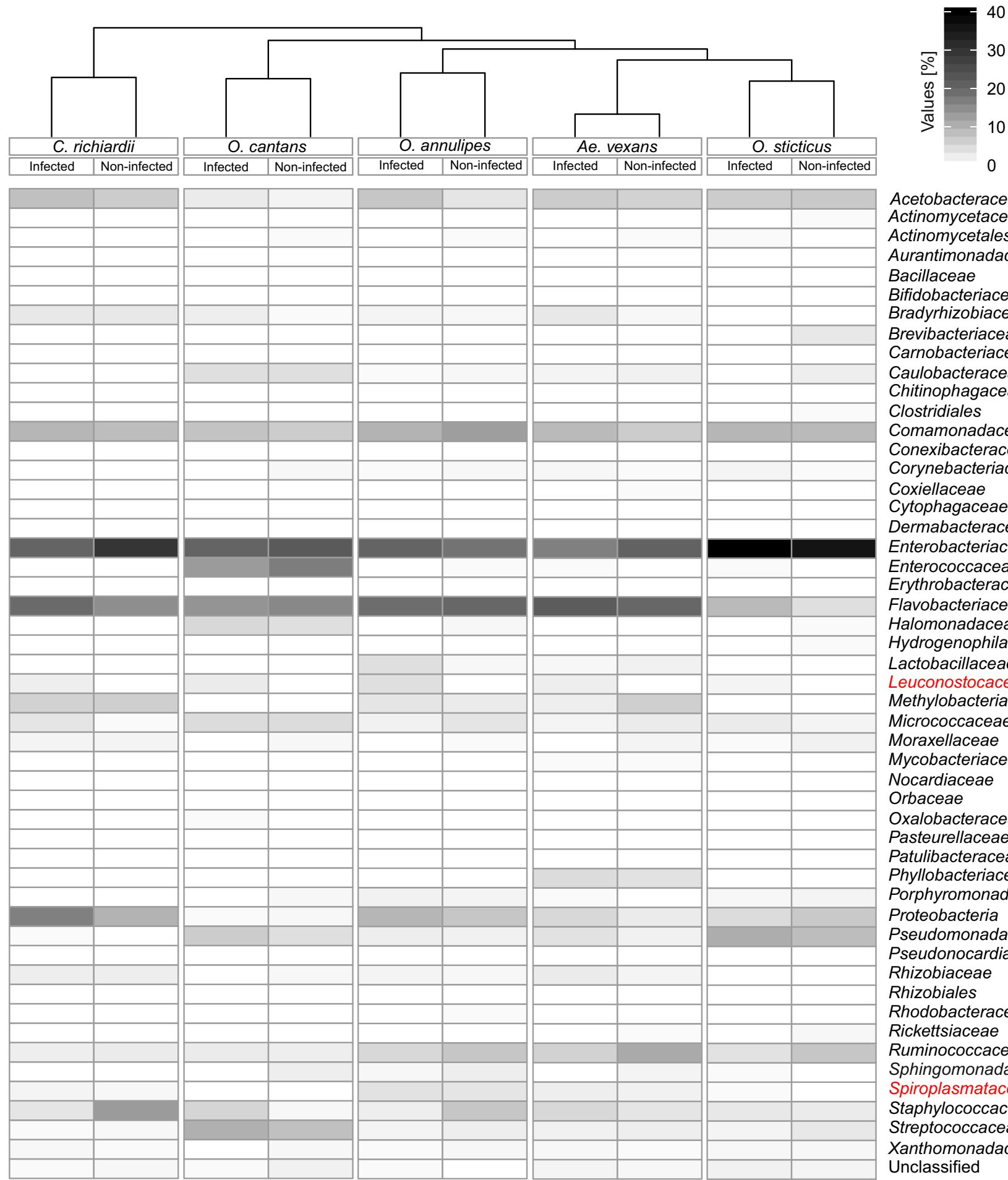

Acetobacteraceae Actinomycetaceae

Actinomycetales

Aurantimonadaceae

Bacillaceae

Bifidobacteriaceae

Bradyrhizobiaceae

Brevibacteriaceae

Carnobacteriaceae

Caulobacteraceae

Chitinophagaceae

Clostridiales

Comamonadaceae

Conexibacteraceae

Corynebacteriaceae

Coxiellaceae

Cytophagaceae

Dermabacteraceae

Enterobacteriaceae

Enterococcaceae

Erythrobacteraceae

Flavobacteriaceae

Halomonadaceae

Hydrogenophilaceae

Lactobacillaceae

Leuconostocaceae

Methylobacteriaceae

Micrococcaceae

Moraxellaceae

Mycobacteriaceae

Nocardiaceae

Orbaceae

Oxalobacteraceae

Pasteurellaceae

Patulibacteraceae

Phyllobacteriaceae

Porphyromonadaceae

Proteobacteria

Pseudomonadaceae

Pseudonocardiaceae

Rhizobiaceae

Rhizobiales

Rhodobacteraceae

Rickettsiaceae

Ruminococcaceae

Sphingomonadaceae

Spiroplasmataceae

Staphylococcaceae

Streptococcaceae

Xanthomonadaceae

Unclassified

Values [\%]

$\begin{array}{lllll}0 & 1 & 2 & 3 & 4\end{array}$

Leuconostocaceae

Spiroplasmataceae

Fig. 1 Heatmap of hierarchical clustering of family-level bacterial microbiome composition profiles. Darker colour represents higher abundance in mosquitoes' gut microbiota. ZOTUs not assigned to families were grouped into higher taxa 
that may be associated with the presence of microsporidians (Fig. 5). The first one included 35 microbiota found in microsporidian-infected mosquitoes, which represented all sampled mosquito species, and one microbiome detected in non-infected $O$. cantans individual. The second group consisted mostly of microbiota found in non-infected mosquitoes, also represented by all of the species. However, nine microbiota of infected individuals, including three each of $A$ e. vexans, $O$. annulipes and $O$. cantans, also belonged to this group. The last group contained both infected and noninfected mosquitoes.

The microbiota of mosquitoes infected with microsporidians had a predicted metabolism more directed to the biosynthesis of ansamycins $\left(p<1 \mathrm{e}^{-15}\right)$, the biosynthesis of vancomycin group antibiotics $\left(p<1 \mathrm{e}^{-15}\right)$ and the pentose phosphate pathway $\left(p<1 \mathrm{e}^{-15}\right)$, when compared to the microbiome of non-infected mosquitoes (Fig. 6). The proportion of these pathways relative to all pathways detected in infected mosquitoes was $8.0 \%(\mathrm{SD}=2.5 \%)$ and $5.5 \%$ $(\mathrm{SD}=2.3 \%)$ in non-infected ones. The difference in mean proportion between these pathways ranged from $0.5 \%$ for the pentose phosphate pathway to $1.2 \%$ for the biosynthesis pathways of ansamycins.

\section{Discussion}

\section{General Structure of Bacterial Communities}

Our 16S rRNA microbial profiling results show that gut microbiota in field-collected mosquitoes sampled in Poland were dominated by Proteobacteria (about 60\%) and Bacteroidetes and Firmicutes (each of about 20\%) These results are broadly consistent with the findings of a largescale study conducted in South Korea in which the gut bacteria of 305 insects, including mosquitoes, were examined [76]. Similarly, high contributions of Proteobacteria and Bacteroidetes were also found in the gut microbiota of insects sampled in Honolulu, Hawaii [77]. Moreover, we observed that the Comamonadaceae, Enterobacteriaceae, Flavobacteriaceae and Pseudomonadaceae are the largest contributors to the gut microbiota of both the infected and non-infected mosquitoes. The representatives of these families have also been identified during previous studies concerning mosquito microbiota [78-80].

The mosquito gut microbiome composition depends on factors that mainly affect the larval stages, such as environmental microbes, feeding behaviours, or biotic agents [81]. Mosquito larvae acquire bacteria during the feedingfiltering process or by vertical transmission [82-85]. It has been demonstrated that bacteria that have colonised the larvae are later found in adult individuals [83, 86, 87].
However, some evidence suggests that the habitat shift from aquatic to terrestrial may decrease the gut bacterial diversity [88, 89]. Moreover, diet amount and type, such as flower nectar, honeydew, fruits or blood, may also affect the adult mosquito microbiome structure. For example, in Ae. aegypti, a high diet abundance was positively correlated with Enterobacteriaceae and Flavobacteriaceae and negatively with Sphingomonadaceae [90]. The change in the microbiome composition as a result of the diet change was also observed in An. gambiae: bacteria belonging to the SAR11 clade were abundant in sugar-fed mosquitoes, while an increased presence of Enterobacteriaceae, Yersiniaceae and Pseudomonadaceae was noticed in mosquitoes after blood feeding [91]. However, most of the studies mentioned above focused on selected mosquito species and were conducted on laboratory-bred populations.

Reports on whether the mosquito microbiome composition can be species-dependent are sparse. Our ANCOM and UPGMA analyses suggest that adults in natural populations host microbial communities that vary among mosquito species. Similar observations have been provided for other Culicidae species, such as Ae. japonicus, Ae. triseriatus, Culex coronator, $C x$. nigripalpus and $C x$. restuans [81, 87]. It is well recognised that species specificity of gut microbiota has a biological basis, and some mechanisms underlying reciprocal host-bacteria selection have already been proposed (for review see [92]); however, future studies are needed because these reports are scarce and mainly involve vertebrates.

Although PCoA considering all mosquito microbiota as one pooled sample suggested that microsporidians do not affect the gut bacterial compositions, the analyses carried out for each species separately grouped mosquito microbiota according to the infection. An exception was $O$. cantans, for which we observed a partial overlap between the microbial compositions of infected and microsporidianfree individuals. Further studies, including quantitative analyses, should be performed to determine whether this result is associated with the infecting microsporidian species or the level of infestation.

\section{Gut Microbiome Members Associated with Microsporidian Infection}

Our data show that infection by microsporidians is associated with a change in the gut microbial composition of the host. To our knowledge, no prior studies have examined the impact of microsporidian infections on the mosquito microbiota, while reports about correlations between microsporidians and microbiota of other invertebrate hosts are sparse and mainly concern honeybees. For instance, it has been noted that the abundance of Alphaproteobacteria decreased in Ap. mellifera honey bees infected with $N$. 


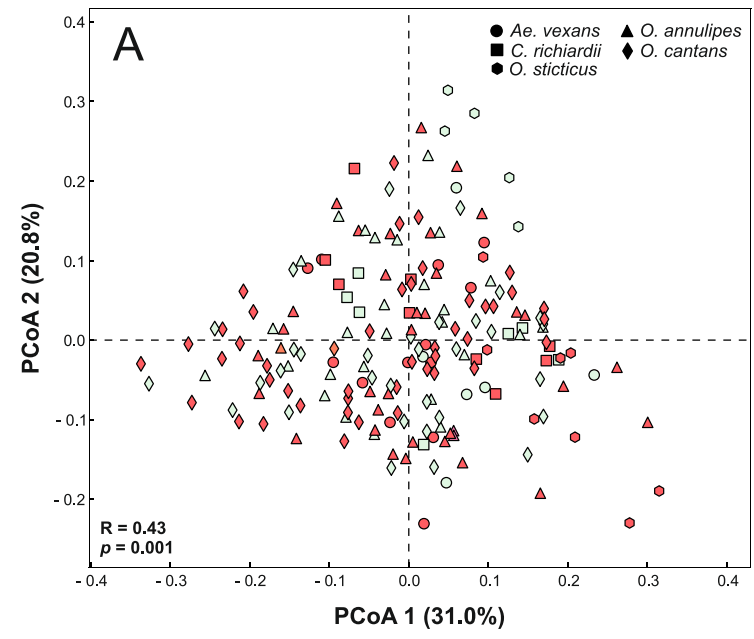

All mosquitoes

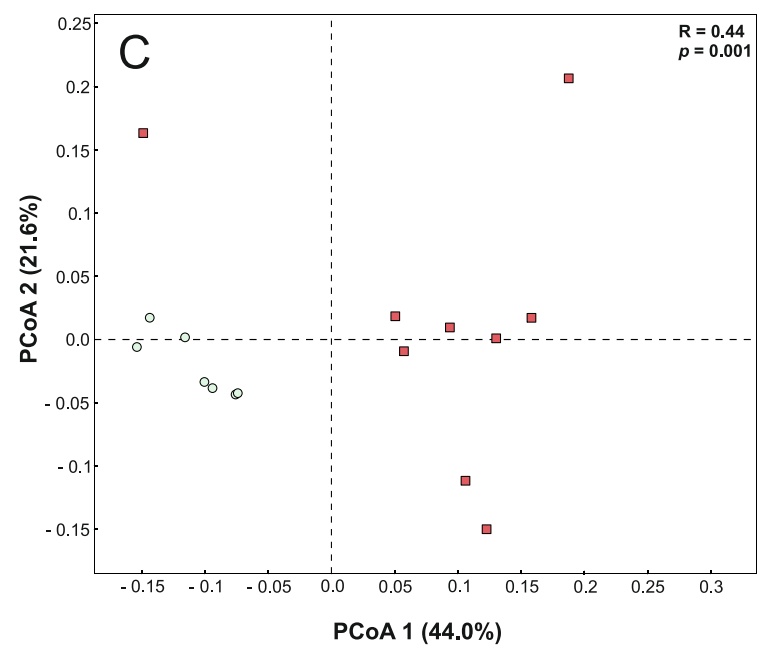

Coquillettidia richiardii

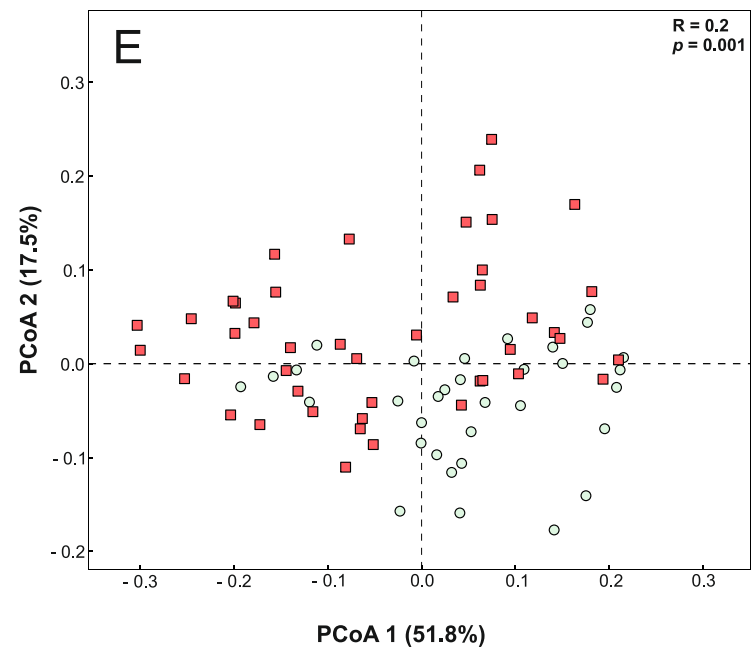

Ochlerotatus cantans

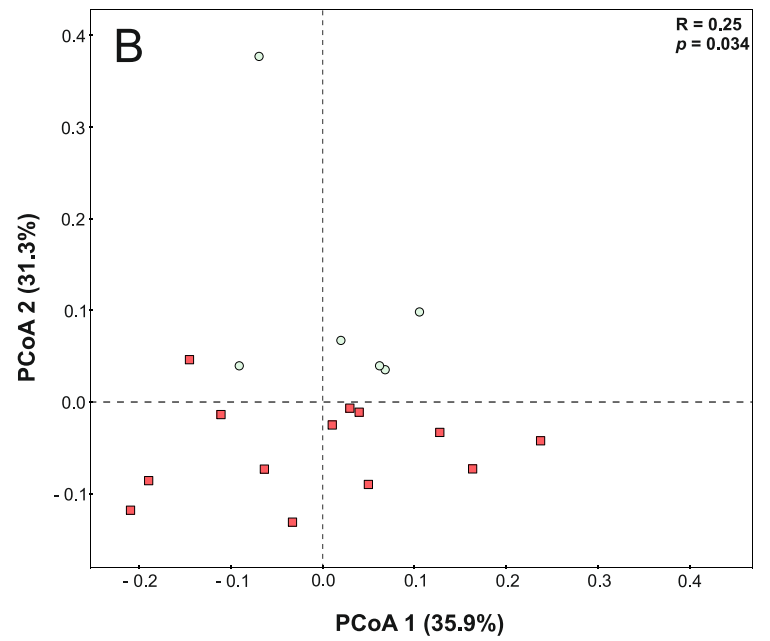

Aedes vexans

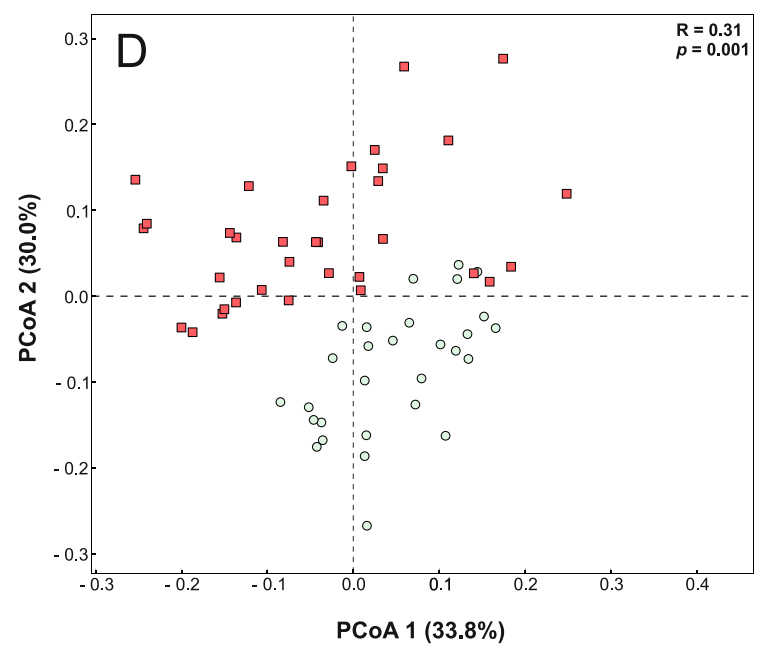

Ochlerotatus annulipes

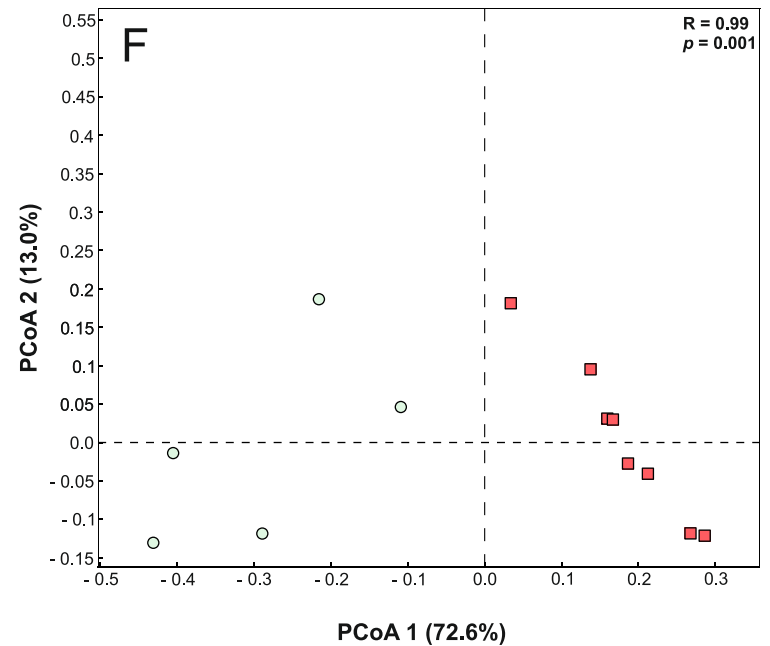

Ochlerotatus sticticus

Infected Non-infected 
४Fig. 2 Gut microbiome structure differentiation and inter-individual similarity based on identified bacterial species between (A) all mosquitoes, (B) Aedes vexans, (C) Coquillettidia richiardii, (D) Ochlerotatus annulipes, (E) O. cantans and (F) O. sticticus. Mosquito species are categorised as infected (red squares) or non-infected (green circles). The correlation between microbiota composition and microsporidian infection $(\mathbf{B}-\mathbf{F})$ and between microbiota composition and both mosquito species and microsporidian infection (A) was tested using the analysis of similarities (ANOSIM) with 999 permutations. $R$ value indicates the ANOSIM statistic which compares the mean of ranked dissimilarities between groups to the mean of ranked dissimilarities within groups, while $p$-value is statistically significant

ceranae and that this effect was stronger when the infected bees were chemically exposed to insecticides or fungicides; additionally, the Gammaproteobacteria abundance increased in the Nosema-infected bees, but significantly only when hosts were co-exposed to pesticides [93]. Our results are partially consistent with these findings. We also noted the decreased abundance of Alphaproteobacteria in microsporidian-infected $C o$. richiardii, $O$. cantans and $O$. sticticus. In addition, we found an increased contribution of Gammaproteobacteria in infected $O$. annulipes and $O$. sticticus.

Our results suggest that microsporidian infections are correlated with specific gut microbiome members. The occurrence of $W$. cf. viridescens was unequivocally correlated with infection. The genus Weissella belongs to lactic acid bacteria [94-96]. Although they are somewhat ubiquitous in the environment, such as soil, lakes and sediments of a coastal marsh [94-96], Weissella spp. are also found as components of insect gut microbiota, for example, of Hymenoptera and Orthoptera [16, 97]. Moreover, it has been shown that microsporidians may reduce the $\mathrm{pH}$ in the gut, inhibiting the growth of most bacteria, except for acid-tolerant species [16]. Our results concur with these observations because Weissella spp. can survive in an acidic environment [16, 94-96].

The occurrence of Spiroplasma sp. PL03 and W. cf. viridescens in microsporidia-positive mosquitoes suggests associations between these bacteria and parasitic infection. Spiroplasma is broadly distributed among invertebrate hosts, such as insects and spiders [98-101]. Additionally, some endosymbiotic Spiroplasma spp. in insects can confer resistance to a range of parasites, including fungi, nematodes and parasitoids [98-100]. Although most members of the genus are endosymbionts, some species can be strongly virulent in male hosts $[100,101]$. The seven Spiroplasma species isolated, to date, from mosquitoes are not virulent
Table 2 Indicator taxon analysis for infected and non-infected mosquitoes. Bacterial families that are significant indicators of community composition are shown. The "A component" indicates how exclusive the family was for infected or non-infected mosquitoes, where a value of 1 indicates that the family was exclusively found in that group. The "B component" indicates how frequently the given family was found in replicate samples, where 1 means it was found in every sample. The indicator value accounts for both the A and B components together

\begin{tabular}{|c|c|c|c|c|c|}
\hline Infection & Family & A component & B component & Indicator value & $p$-value \\
\hline \multirow[t]{20}{*}{ Non-infected } & Actinomycetaceae & 0.633 & 1 & 0.795 & 0.010 \\
\hline & Aurantimonadaceae & 0.933 & 0.838 & 0.884 & 0.005 \\
\hline & Bacillaceae & 0.733 & 0.913 & 0.818 & 0.005 \\
\hline & Brevibacteriaceae & 0.480 & 0.800 & 0.620 & 0.025 \\
\hline & Conexibacteraceae & 0.934 & 0.538 & 0.708 & 0.010 \\
\hline & Coxiellaceae & 0.680 & 0.925 & 0.793 & 0.005 \\
\hline & Cytophagaceae & 0.932 & 0.125 & 0.341 & 0.030 \\
\hline & Dermabacteraceae & 0.936 & 0.450 & 0.649 & 0.005 \\
\hline & Erythrobacteraceae & 0.885 & 0.400 & 0.595 & 0.010 \\
\hline & Halomonadaceae & 0.854 & 1 & 0.924 & 0.005 \\
\hline & Hydrogenophilaceae & 0.901 & 0.888 & 0.894 & 0.005 \\
\hline & Methylobacteriaceae & 0.588 & 1 & 0.767 & 0.015 \\
\hline & Moraxellaceae & 0.682 & 1 & 0.826 & 0.005 \\
\hline & Mycobacteriaceae & 0.597 & 1 & 0.773 & 0.025 \\
\hline & Orbaceae & 0.606 & 0.850 & 0.718 & 0.005 \\
\hline & Pseudonocardiaceae & 0.561 & 0.913 & 0.715 & 0.015 \\
\hline & Rhodobacteraceae & 0.825 & 0.775 & 0.799 & 0.005 \\
\hline & Rickettsiaceae & 0.853 & 0.825 & 0.839 & 0.005 \\
\hline & Ruminococcaceae & 0.607 & 0.938 & 0.754 & 0.005 \\
\hline & Sphingomonadaceae & 0.742 & 1 & 0.861 & 0.005 \\
\hline \multirow[t]{7}{*}{ Infected } & Acetobacteraceae & 0.610 & 1 & 0.781 & 0.005 \\
\hline & Bradyrhizobiaceae & 0.629 & 1 & 0.793 & 0.005 \\
\hline & Leuconostocaceae & 1 & 0.139 & 0.373 & 0.005 \\
\hline & Patulibacteraceae & 0.640 & 0.843 & 0.734 & 0.020 \\
\hline & Pseudomonadaceae & 0.609 & 1 & 0.780 & 0.010 \\
\hline & Rhizobiaceae & 0.623 & 1 & 0.789 & 0.005 \\
\hline & Spiroplasmataceae & 0.616 & 0.926 & 0.755 & 0.005 \\
\hline
\end{tabular}




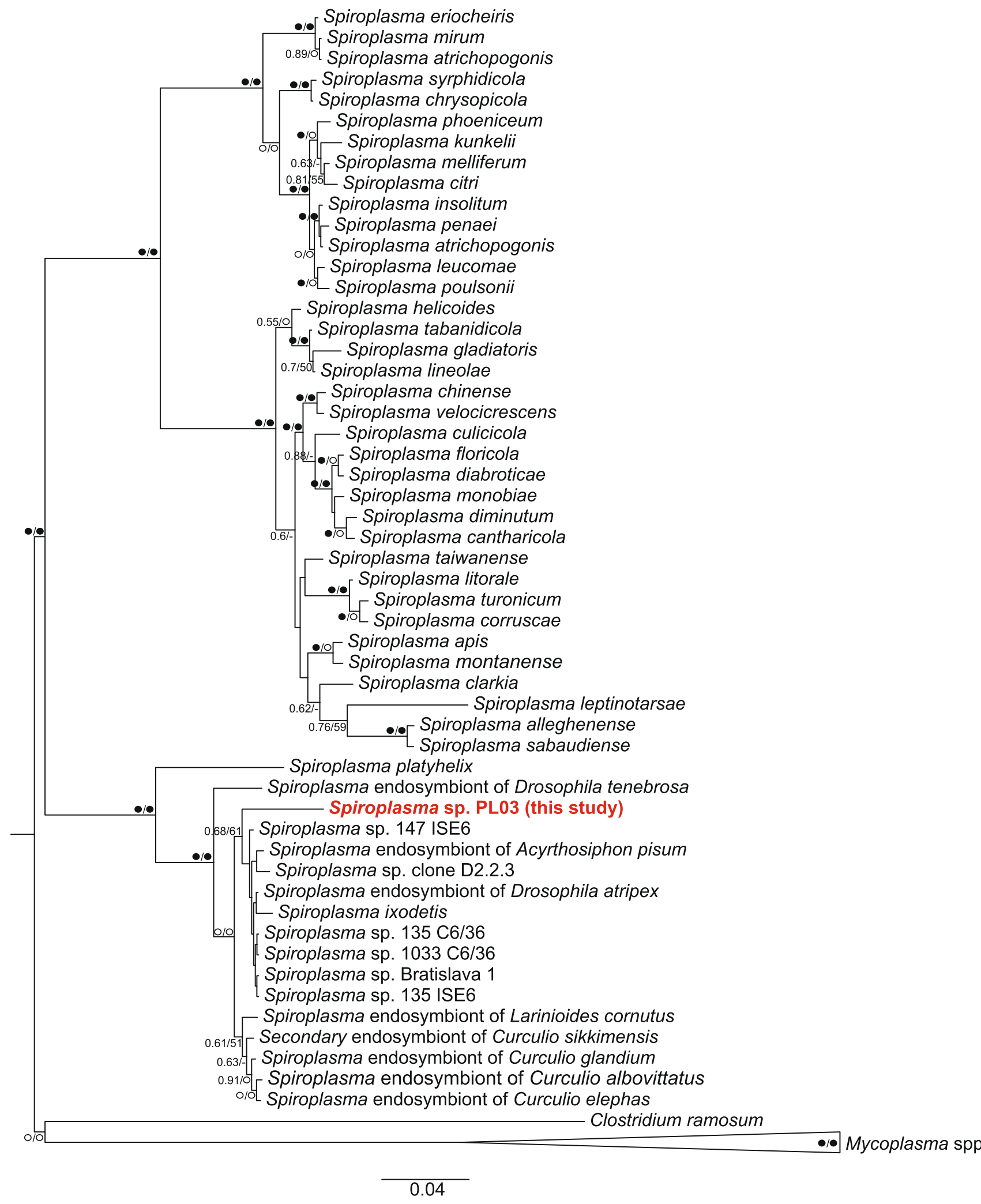

Fig. 3 Phylogenetic tree of Spiroplasma 16S rRNA gene sequences inferred from BI and ML analyses. Values near branches show Bayesian posterior probabilities (PP) and bootstrap support values (BS)
(PP/BS). Black circles: maximally supported; empty circles: supported $>0.95 \mathrm{PP}$ and $>75 \%$ BS. Sequence found in this study is in red 


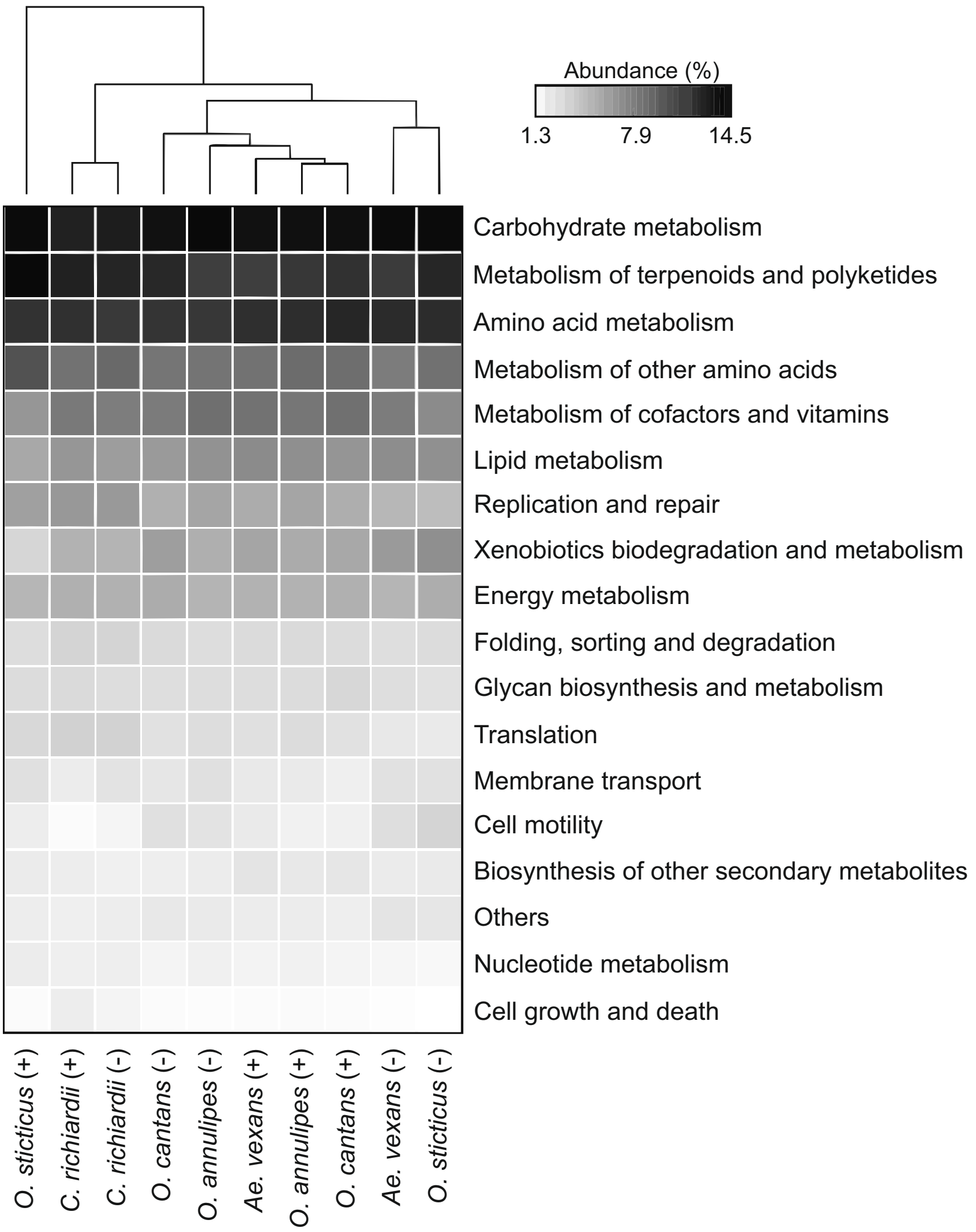

Fig. 4 Heatmap of hierarchical clustering of relative abundance of the predicted pathways of the metagenome related to KEGG at level 2. Darker colour represents higher abundance in mosquito gut microbiome. Mosquito species are categorised as infected (+) or non-infected (-) 
Fig. 5 Pathways' structure differentiation and inter-individual similarity based on the predicted pathways of the metagenome. PCoA was used to show patterns of separation. Point colour shows infection (red: infected; green: non-infected), and point shape identifies mosquito species (circle: Aedes vexans, square: Coquillettidia richiardii, triangle: Ochlerotatus annulipes, diamond: $O$. cantans, hexagon: $O$. sticticus). Ellipses shows groups of mosquitoes associated with the presence of microsporidians (yellow: mosquitoes associated with the presence of microsporidians, blue: mosquitoes not associated with the presence of microsporidians, green: both infected and non-infected mosquitoes)
Infected $\square$ Non-infected

- Ae. vexans $\Delta$ O. annulipes O. sticticus

C. richiardii $\diamond$. cantans

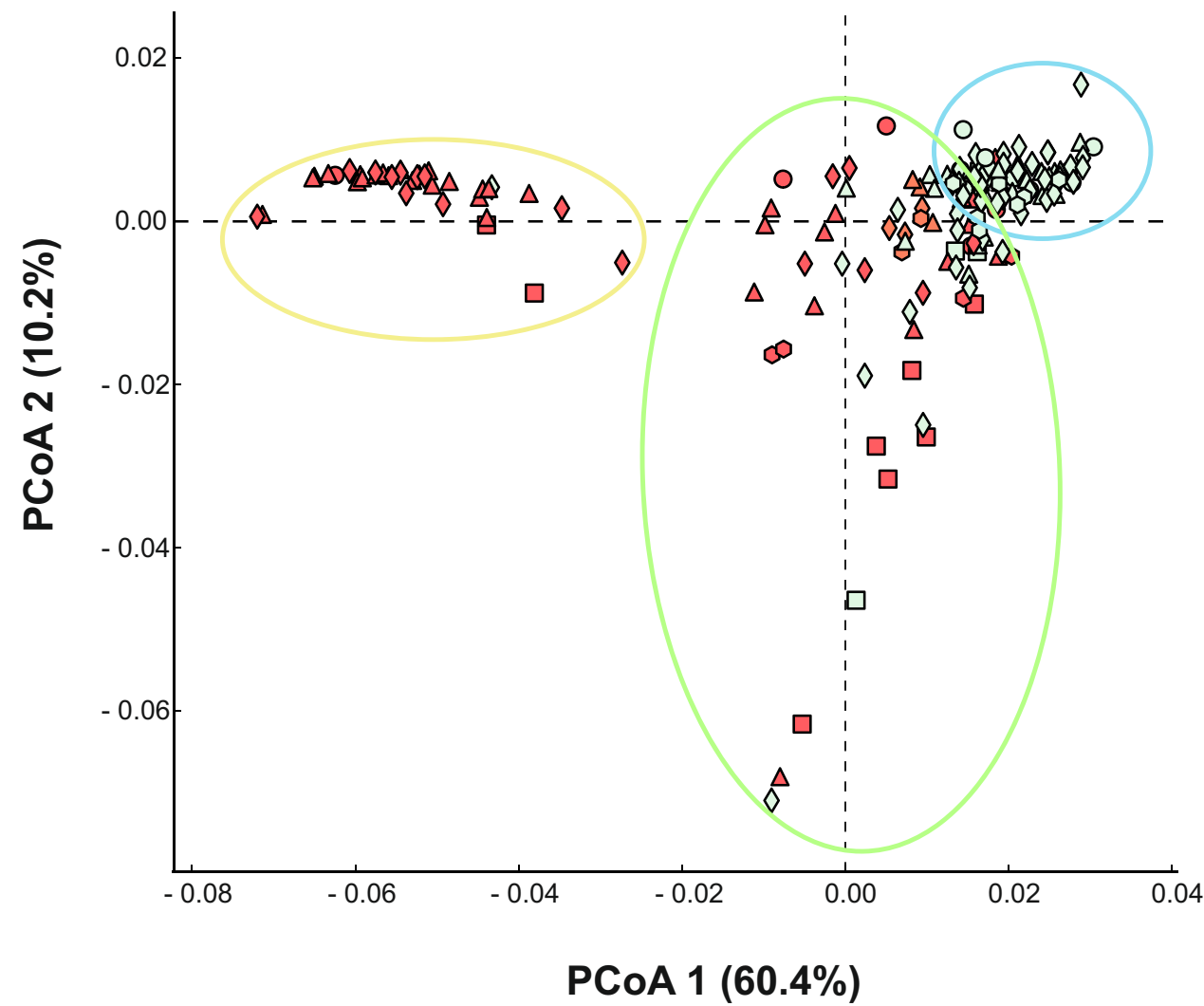

to mosquitoes (Supplementary Table 10). Therefore, greater participation of Spiroplasma sp. PL03 in microsporidianpositive mosquitoes than in non-infected ones suggests an association of these bacteria with microsporidian infection. Our phylogenetic analyses confirmed that the identified Spiroplasma sp. PL03 is an endosymbiotic species; however, many questions remain unresolved, such as transmission mode, tissue tropism and fitness effects [98].

\section{Functional Analysis of Mosquito Microbiota During Microsporidian Infection}

The prediction of metagenome functional content from the $16 \mathrm{~S}$ rRNA gene sequencing suggests that during microsporidian infection, the mosquito microbiome is relatively more abundant in bacterial species capable of metabolising terpenoids and polyketides and synthesising ansamycin and vancomycin group antibiotics. The oxidative phosphorylation and pentose phosphate pathways are also comparatively more abundant in the microbiota of infected hosts.

The increased ability to synthesise antibiotics appears to be a natural mechanism to protect the host already weakened by parasitic infection. Ansamycins form a class of bacterial macrocyclic polyketides that exhibit broad inhibitory activities, including antibacterial, antifungal, antiviral and immunosuppressant [102, 103]. For example, rifamycin, a naphthalenic ansamycin, inhibits RNA transcription in many bacterial species [104, 105]. Vancomycin is a branched tricyclic glycopeptide antibiotic that inhibits cell wall synthesis and is active against most Gram-positive bacteria, including anaerobic clostridia [106, 107]. Weissella cf. viridescens belongs to the family Leuconostocaceae, whose members are intrinsically vancomycin-resistant [94, 95, 108]. Therefore, the vancomycin synthesis could explain the presence of Weissella only in microsporidian-infected mosquitoes.

Microsporidians have lost most of the genes needed for making primary metabolites, such as nucleotides and amino acids, and have a limited capacity to generate adenosine triphosphate (ATP) [16, 109, 110]. The loss of key pathways for energy generation has resulted in microsporidians having to obtain those substrates from host cells [111]. Moreover, He et al. [109] showed that after the induction of spore germination, the majority of the microsporidian genes involved in the pentose phosphate pathway are downregulated. Thus, they suggested that sporoplasm might inhibit its carbon metabolic activity and obtain the substances required for 


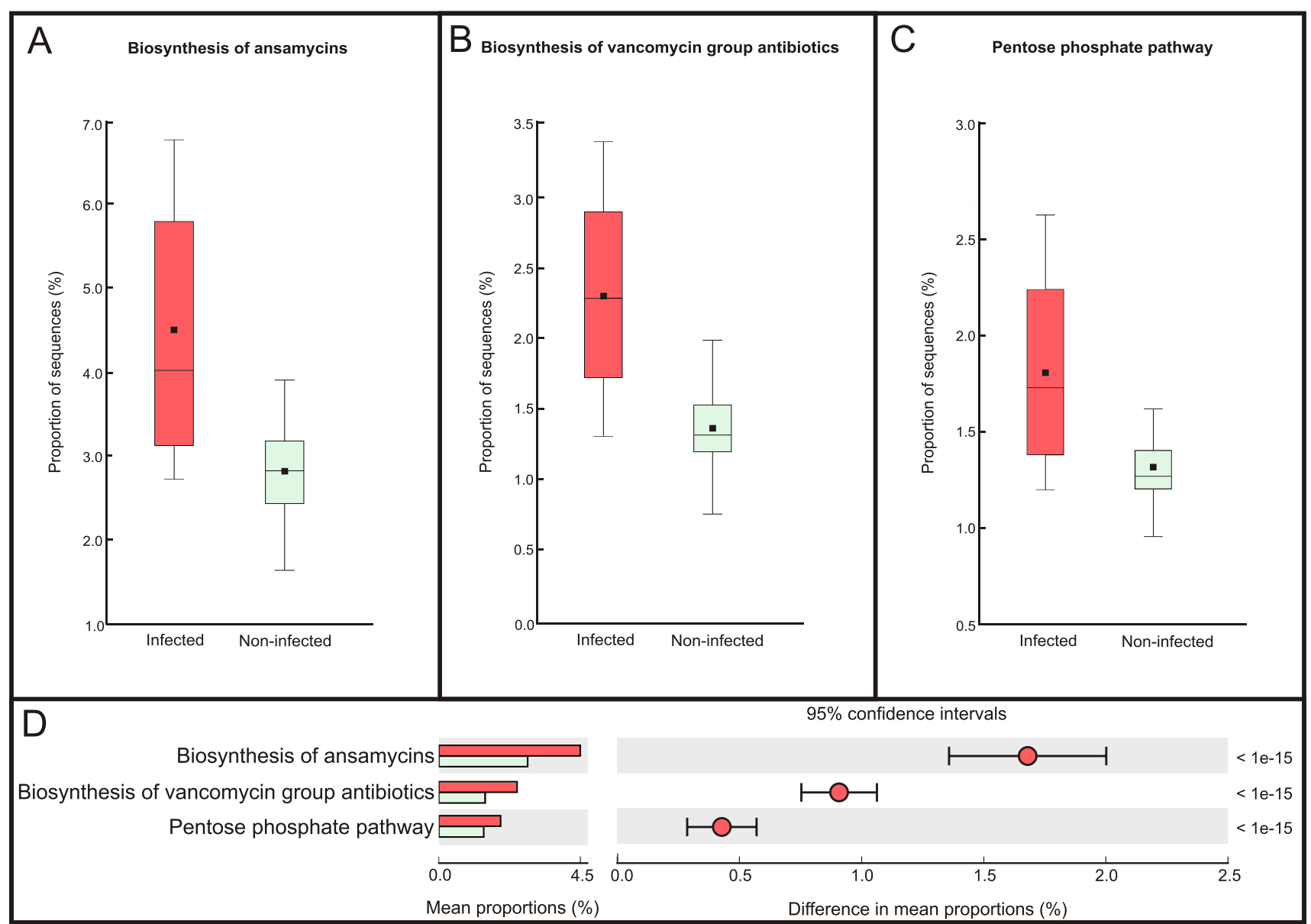

Fig. 6 KEGG pathways at level 3 for the infected and non-infected mosquitoes by Microsporidia. Box plots of (A) biosynthesis of ansamycins, (B) biosynthesis of vancomycin group antibiotics, (C)

proliferation from host cells. Our observation that the pentose phosphate pathways are relatively more abundant in the microbiota of microsporidian-infected mosquitoes supports the hypothesis that microsporidians might manipulate biological processes in their environment to promote nucleotide synthesis and maximise the potential for ATP and nucleotide import [13, 109]. However, we acknowledge that our results cannot resolve whether the predicted observed alteration in gut microbial functionalities is a microsporidian-mediated response or caused by the host immune system or due to a change in the mycobiome. Therefore, future studies should be carried out, including mycobiome identification and host transcriptome analysis.

\section{Conclusion}

In this paper, we suggest that microsporidian infection shapes the microbial community structure and microbial activity in infected mosquitoes, especially the biosynthesis pentose phosphate pathway and (D) extended error bar plot indicating differences in functional profiles

of antibiotics and the pentose phosphate pathway. This result confirms previous findings that parasites modulate the gut microbiome in insects and that this is an important agent during modulated host- and parasite-associated microbiome interactions. Endosymbiotic Spiroplasma sp. PL03 and $W$. cf. viridescens, two bacterial species found in this study, represent bacteria whose participation in the mosquito gut is highly dependent on microsporidian infection. Our results imply that the gut microbiome may also affect pathogens vectored by mosquitoes. For example, the previously observed resistance of microsporidian-infected mosquitoes to Plasmodium transmission [10] might be related to changes in the structure and metabolism of the mosquito microbiome. Considering that the oocysts must nest in the gut, settled beneath the epithelium, and attached to the basal lamina, the alterations in the intestinal microbiome caused by microsporidian infection cannot be excluded as one of the mechanisms preventing the parasite's development. However, more extensive research, including the determination of metabolic activity based 
on quantitative methods and experimental infection under controlled conditions, should be carried out to test this hypothesis.

Supplementary Information The online version contains supplementary material available at https://doi.org/10.1007/s00248-021-01944-z.

Acknowledgements This study was supported by grant no. 2020/37/N/ NZ8/01735 from the National Science Centre of Poland and the Initiative of Excellence - Research University (05/IDUB/2019/94) at Adam Mickiewicz University, Poznan, Poland. This research was supported in part by PLGrid Infrastructure.

\section{Declarations}

Conflict of Interest The authors declare no competing interests.

Open Access This article is licensed under a Creative Commons Attribution 4.0 International License, which permits use, sharing, adaptation, distribution and reproduction in any medium or format, as long as you give appropriate credit to the original author(s) and the source, provide a link to the Creative Commons licence, and indicate if changes were made. The images or other third party material in this article are included in the article's Creative Commons licence, unless indicated otherwise in a credit line to the material. If material is not included in the article's Creative Commons licence and your intended use is not permitted by statutory regulation or exceeds the permitted use, you will need to obtain permission directly from the copyright holder. To view a copy of this licence, visit http://creativecommons.org/licenses/by/4.0/.

\section{References}

1. Stentiford GD, Becnel JJ, Weiss LM et al (2016) Microsporidia - emergent pathogens in the global food chain. Trends Parasitol 32:336-348. https://doi.org/10.1016/j.pt.2015.12.004

2. Han B, Pan G, Weiss LM (2021) Microsporidiosis in humans. Clin Microbiol Rev. https://doi.org/10.1128/CMR.00010-20

3. Becnel JJ, Andreadis TG (2014) Microsporidia in insects. In: Weiss LM, Becnel JJ (eds) Microsporidia. John Wiley \& Sons Inc, Chichester, pp 521-570

4. Andreadis TG (2007) Microsporidian parasites of mosquitoes. J Am Mosq Control Assoc 23:3-29. https://doi.org/10.2987/8756971X(2007)23[3:MPOM]2.0.CO;2

5. Becnel JJ, Takvorian PM, Cali A (2014) Checklist of available generic names for microsporidia with type species and type hosts. In: Weiss LM, Becnel JJ (eds) Microsporidia. John Wiley \& Sons Inc, Chichester, pp 671-686

6. Aliota MT, Chen CC, Dagoro H et al (2011) Filarial worms reduce plasmodium infectivity in mosquitoes. PLoS Negl Trop Dis 5:1-14. https://doi.org/10.1371/journal.pntd.0000963

7. Deng S, Huang Q, Wei H et al (2004) (2019) Beauveria bassiana infection reduces the vectorial capacity of Aedes albopictus for the Zika virus. J Pest Sci 92:781-789. https://doi.org/10.1007/ s10340-019-01081-0

8. Moreira LA, Iturbe-Ormaetxe I, Jeffery JA et al (2009) A Wolbachia symbiont in Aedes aegypti limits infection with dengue, chikungunya, and plasmodium. Cell 139:1268-1278. https://doi. org/10.1016/j.cell.2009.11.042

9. Jupatanakul N, Sim S, Dimopoulos G (2014) The insect microbiome modulates vector competence for arboviruses. Viruses 6:4294-4313. https://doi.org/10.3390/v6114294
10. Herren JK, Mbaisi L, Mararo E et al (2020) A microsporidian impairs Plasmodium falciparum transmission in Anopheles arabiensis mosquitoes. Nat Commun 11:2187. https://doi.org/10. 1038/s41467-020-16121-y

11. Bargielowski I, Koella JC (2009) A possible mechanism for the suppression of Plasmodium berghei development in the mosquito Anopheles gambiae by the Microsporidian Vavraia culicis. PLoS ONE 4:e4676. https://doi.org/10.1371/journal.pone.0004676

12. Ang'ang'o LM (2018) Molecular characterisation of microsporidia mb species and correlation with plasmodium presence in anopheles mosquitoes in Mwea and Mbita. University of Nairobi, Kenya

13. Pan G, Bao J, Ma Z et al (2018) Invertebrate host responses to microsporidia infections. Dev Comp Immunol 83:104-113. https://doi.org/10.1016/j.dci.2018.02.004

14. Biganski S, Fückel S, Jehle JA, Kleespies RG (2021) Infection effects of the new microsporidian species Tubulinosema suzukii on its host Drosophila suzukii. Sci Rep 11:10151. https://doi.org/ 10.1038/s41598-021-89583-9

15. Dorhout DL, Sappington TW, Lewis LC, Rice ME (2011) Flight behaviour of European corn borer infected with Nosema pyrausta. J Appl Entomol 135:25-37. https://doi.org/10.1111/j. 1439-0418.2010.01523.x

16. Shi W, Guo Y, Xu C et al (2014) Unveiling the mechanism by which microsporidian parasites prevent locust swarm behavior. Proc Natl Acad Sci 111:1343-1348. https://doi.org/10.1073/ pnas.1314009111

17. Tan SQ, Yin Y, Cao KL et al (2021) Effects of a combined infection with Paranosema locustae and Beauveria bassiana on Locusta migratoria and its gut microflora. Insect Sci 28:347-354. https://doi.org/10.1111/1744-7917.12776

18. Rubanov A, Russell KA, Rothman JA et al (2019) Intensity of Nosema ceranae infection is associated with specific honey bee gut bacteria and weakly associated with gut microbiome structure. Sci Rep 9:1-8. https://doi.org/10.1038/s41598-019-40347-6

19. Dheilly NM, Poulin R, Thomas F (2015) Biological warfare: microorganisms as drivers of host-parasite interactions. Infect Genet Evol 34:251-259. https://doi.org/10.1016/j.meegid.2015. 05.027

20. Louradour I, Monteiro CC, Inbar E et al (2017) The midgut microbiota plays an essential role in sand fly vector competence for Leishmania major. Cell Microbiol 19:1-13. https://doi.org/ 10.1111/cmi.12755

21. Kelly PH, Bahr SM, Serafim TD et al (2017) The gut microbiome of the vector Lutzomyia longipalpis is essential for survival of Leishmania infantum. MBio 8:e01121-16. https://doi.org/10.1128/mBio. 01121-16

22. Fredensborg BL, Fossdal í Kálvalíð I, Johannesen TB et al (2020) Parasites modulate the gut-microbiome in insects: a proof-ofconcept study. PLoS ONE 15:e0227561. https://doi.org/10.1371/ journal.pone.0227561

23. Lee J-H, Lee K-A, Lee W-J (2017) Microbiota, gut physiology, and insect immunity. In: Ligoxygakis P (ed) Advances in Insect Physiology. Elsevier Ltd., pp 111-138. https://doi.org/10.1016/ bs.aiip.2016.11.001

24. Caragata EP, Tikhe CV, Dimopoulos G (2019) Curious entanglements: interactions between mosquitoes, their microbiota, and arboviruses. Curr Opin Virol 37:26-36. https://doi.org/10.1016/j. coviro.2019.05.005

25. Strand MR (2018) Composition and functional roles of the gut microbiota in mosquitoes. Curr Opin Insect Sci 28:59-65. https:// doi.org/10.1016/j.cois.2018.05.008

26. Gao H, Cui C, Wang L et al (2020) Mosquito microbiota and implications for disease control. Trends Parasitol 36:98-111. https://doi.org/10.1016/j.pt.2019.12.001 
27. Capone A, Ricci I, Damiani C et al (2013) Interactions between Asaia, Plasmodium and Anopheles: new insights into mosquito symbiosis and implications in Malaria Symbiotic Control. Parasit Vectors 6:1-13. https://doi.org/10.1186/1756-3305-6-182

28. Bai L, Wang L, Vega-Rodríguez J et al (2019) A gut symbiotic bacterium serratia marcescens renders mosquito resistance to plasmodium infection through activation of mosquito immune responses. Front Microbiol 10:e1580. https://doi.org/10.3389/ fmicb.2019.01580

29. Scolari F, Casiraghi M, Bonizzoni M (2019) Aedes spp. and their microbiota: a review. Front Microbiol 10:e2036. https://doi.org/ 10.3389/fmicb.2019.02036

30. Onchuru TO, Ajamma YU, Burugu M et al (2016) Chemical parameters and bacterial communities associated with larval habitats of Anopheles, Culex and Aedes mosquitoes (Diptera: Culicidae) in western Kenya. Int J Trop Insect Sci 36:146-160. https://doi.org/10.1017/S1742758416000096

31. Coon KL, Brown MR, Strand MR (2016) Mosquitoes host communities of bacteria that are essential for development but vary greatly between local habitats. Mol Ecol 25:5806-5826. https:// doi.org/10.1111/mec. 13877

32. Gendrin M, Rodgers FH, Yerbanga RS et al (2015) Antibiotics in ingested human blood affect the mosquito microbiota and capacity to transmit malaria. Nat Commun 6:1-7. https://doi.org/10. 1038/ncomms6921

33. Guégan M, Minard G, Tran FH et al (2018) Short-term impacts of anthropogenic stressors on Aedes albopictus mosquito vector microbiota. FEMS Microbiol Ecol 94:fiy188. https://doi.org/10. 1093/femsec/fiy 188

34. Wang X, Liu T, Wu Y et al (2018) Bacterial microbiota assemblage in Aedes albopictus mosquitoes and its impacts on larval development. Mol Ecol 27:2972-2985. https://doi.org/10.1111/ mec. 14732

35. Yadav KK, Datta S, Naglot A et al (2016) Diversity of cultivable midgut microbiota at different stages of the asian tiger mosquito, Aedes albopictus from Tezpur. India PLoS One 11:e167409. https://doi.org/10.1371/journal.pone.0167409

36. Muturi EJ, Dunlap C, Ramirez JL et al (2018) Host blood-meal source has a strong impact on gut microbiota of Aedes aegypti. FEMS Microbiol Ecol 95:1-9. https://doi.org/10.1093/femsec/ fiy 213

37. Minard G, Tran FH, Van VT et al (2018) Shared larval rearing environment, sex, female size and genetic diversity shape Ae. albopictus bacterial microbiota. PLoS ONE 13:1-16. https://doi. org/10.1371/journal.pone.0194521

38. Huang W, Wang S, Jacobs-Lorena M (2020) Use of microbiota to fight mosquito-borne disease. Front Genet 11:1-6. https://doi. org/10.3389/fgene.2020.00196

39. Guégan M, Zouache K, Démichel C et al (2018) The mosquito holobiont: fresh insight into mosquito-microbiota interactions. Microbiome 6:49. https://doi.org/10.1186/s40168-018-0435-2

40. Romoli O, Gendrin M (2018) The tripartite interactions between the mosquito, its microbiota and Plasmodium. Parasit Vectors 11:1-8. https://doi.org/10.1186/s13071-018-2784-x

41. Wu P, Sun P, Nie K et al (2019) A gut commensal bacterium promotes mosquito permissiveness to arboviruses. Cell Host Microbe 25:101-112.e5. https://doi.org/10.1016/j.chom.2018. 11.004

42. Bian G, Joshi D, Dong Y et al (2013) Wolbachia invades Anopheles stephensi populations and induces refractoriness to plasmodium infection. Science (80- ) 340:748-751. https://doi.org/10. 1126/science. 1236192

43. Hughes GL, Koga R, Xue P et al (2011) Wolbachia infections are virulent and inhibit the human malaria parasite Plasmodium falciparum in Anopheles Gambiae. PLoS Pathog 7:e1002043. https://doi.org/10.1371/journal.ppat.1002043
44. Trzebny A, Slodkowicz-Kowalska A, Becnel JJ et al (2020) A new method of metabarcoding microsporidia and their hosts reveals high levels of microsporidian infections in mosquitoes (Culicidae). Mol Ecol Resour 20:1486-1504. https://doi.org/10. 1111/1755-0998.13205

45. Makowska N, Philips A, Dabert M et al (2020) Metagenomic analysis of $\beta$-lactamase and carbapenemase genes in the wastewater resistome. Water Res 170:115277. https://doi.org/10. 1016/j.watres.2019.115277

46. Bolger AM, Lohse M, Usadel B (2014) Trimmomatic: a flexible trimmer for Illumina sequence data. Bioinformatics 30:21142120. https://doi.org/10.1093/bioinformatics/btu170

47. Hannon GJ (2010) FASTX-Toolkit. In: http://hannonlab.cshl.edu. Accessed Aug 2020

48. Edgar RC (2013) UPARSE: highly accurate OTU sequences from microbial amplicon reads. Nat Methods 10:996-998. https://doi. org/10.1038/nmeth.2604

49. Edgar RC (2016) UCHIME2 : improved chimera prediction for amplicon sequencing. bioRxiv 074252. https://doi.org/10.1101/ 074252

50. Edgar RC (2016) SINTAX: a simple non-Bayesian taxonomy classifier for 16S and ITS sequences. bioRxiv 074161. https:// doi.org/10.1101/074161

51. Edgar RC, Flyvbjerg H (2018) Octave plots for visualizing diversity of microbial OTUs. bioRxiv 389833. https://doi.org/10.1101/ 389833

52. Edgar RC (2018) Taxonomy annotation and guide tree errors in 16S rRNA databases. PeerJ 2018:e5030. https://doi.org/10.7717/ peerj. 5030

53. Edgar RC (2018) Accuracy of taxonomy prediction for $16 \mathrm{~S}$ rRNA and fungal ITS sequences. PeerJ 2018:1-29. https://doi.org/10. 7717/peerj.4652

54. Yilmaz P, Parfrey LW, Yarza P et al (2014) The SILVA and "all-species Living Tree Project (LTP)" taxonomic frameworks. Nucleic Acids Res 42:643-648. https://doi.org/10.1093/nar/ gkt1209

55. Quast C, Pruesse E, Yilmaz P et al (2013) The SILVA ribosomal RNA gene database project: Improved data processing and webbased tools. Nucleic Acids Res 41:590-596. https://doi.org/10. 1093/nar/gks 1219

56. Glöckner FO, Yilmaz P, Quast C et al (2017) 25 years of serving the community with ribosomal RNA gene reference databases and tools. J Biotechnol 261:169-176. https://doi.org/10.1016/j. jbiotec.2017.06.1198

57. Edgar RC (2018) UNCROSS2: Identification of cross-talk in 16S rRNA OTU tables. bioRxiv 400762. https://doi.org/10.1101/ 400762

58. Douglas GM, Maffei VJ, Zaneveld JR et al (2020) PICRUSt2 for prediction of metagenome functions. Nat Biotechnol 38:685688. https://doi.org/10.1038/s41587-020-0548-6

59. Kanehisa M (2019) Toward understanding the origin and evolution of cellular organisms. Protein Sci 28:1947-1951. https://doi. org/10.1002/pro.3715

60. Yi Y, Fang Y, Wu K et al (2020) Comprehensive gene and pathway analysis of cervical cancer progression. Oncol Lett 19:33163332. https://doi.org/10.3892/ol.2020.11439

61. Kanehisa M, Furumichi M, Sato Y et al (2021) KEGG: integrating viruses and cellular organisms. Nucleic Acids Res 49:D545D551. https://doi.org/10.1093/nar/gkaa970

62. Langille MGI, Zaneveld J, Caporaso JG et al (2013) Predictive functional profiling of microbial communities using 16S rRNA marker gene sequences. Nat Biotechnol 31:814-821. https://doi. org/10.1038/nbt.2676

63. Parks DH, Tyson GW, Hugenholtz P, Beiko RG (2014) STAMP: statistical analysis of taxonomic and functional profiles. 
Bioinformatics 30:3123-3124. https://doi.org/10.1093/bioin formatics/btu494

64. Jang J, Kim B, Lee J et al (2002) Identification of Weissella species by the genus-specific amplified ribosomal DNA restriction analysis. FEMS Microbiol Lett 212:29-34. https://doi.org/10. 1016/S0378-1097(02)00680-8

65. Zhang Z, Schwartz S, Wagner L, Miller W (2000) A greedy algorithm for aligning DNA sequences. J Comput Biol 7:203-214. https://doi.org/10.1089/10665270050081478

66. Morgulis A, Coulouris G, Raytselis Y et al (2008) Database indexing for production MegaBLAST searches. Bioinformatics 24:1757-1764. https://doi.org/10.1093/bioinformatics/btn322

67. Katoh K, Misawa K, Kuma K, Miyata T (2002) MAFFT: a novel method for rapid multiple sequence alignment based on fast Fourier transform. Nucleic Acids Res 30:3059-3066. https://doi.org/ 10.1093/nar/gkf436

68. Lanfear R, Calcott B, Ho SYW, Guindon S (2012) PartitionFinder: combined selection of partitioning schemes and substitution models for phylogenetic analyses. Mol Biol Evol 29:16951701. https://doi.org/10.1093/molbev/mss020

69. Zwickl DM (2006) Genetic algorithm approaches for the phylogenetic analysis of large biological sequence datasets under the maximum likelihood criterion. Dissertation 1-125. The University of Texas at Austin

70. Ronquist F, Teslenko M, Van Der Mark P et al (2012) Mrbayes 3.2: efficient bayesian phylogenetic inference and model choice across a large model space. Syst Biol 61:539-542. https://doi. org/10.1093/sysbio/sys029

71. Rambaut A (2018) FigTree v. 1.4.4. https://github.com/rambaut/ figtree. Accessed Aug 2020

72. R Core Team (2020) R: a language and environment for statistical computing. R Found Stat Comput. Vienna, Austria. https:// www.R-project.org

73. Hothorn T, Hornik K, Wiel MA van de, Zeileis A (2008) Implementing a class of permutation tests: the coin Package. J Stat Softw 28:1-23. https://doi.org/10.18637/jss.v028.i08

74. Clarke KR (1993) Non-parametric multivariate analyses of changes in community structure. Aust J Ecol 18:117-143. https:// doi.org/10.1111/j.1442-9993.1993.tb00438.x

75. De Cáceres M, Legendre P (2009) Associations between species and groups of sites: Indices and statistical inference. Ecology 90:3566-3574. https://doi.org/10.1890/08-1823.1

76. Yun JH, Roh SW, Whon TW et al (2014) Insect gut bacterial diversity determined by environmental habitat, diet, developmental stage, and phylogeny of host. Appl Environ Microbiol 80:5254-5264. https://doi.org/10.1128/AEM.01226-14

77. Jones RT, Sanchez LG, Fierer N (2013) A cross-taxon analysis of insect-associated bacterial diversity. PLoS ONE 8:e61218. https://doi.org/10.1371/journal.pone.0061218

78. Caragata EP, Otero LM, Tikhe CV et al (2021) Microbial diversity of adult Aedes aegypti and water collected from different mosquito aquatic habitats in Puerto Rico. Microb Ecol. https:// doi.org/10.1007/s00248-021-01743-6

79. Linenberg I, Christophides GK, Gendrin M (2016) Larval diet affects mosquito development and permissiveness to Plasmodium infection. Sci Rep 6:1-10. https://doi.org/10.1038/srep38230

80. Vilanova C, Baixeras J, Latorre A, Porcar M (2016) The generalist inside the specialist: gut bacterial communities of two insect species feeding on toxic plants are dominated by Enterococcus sp. Front Microbiol 7:e1005. https://doi.org/10.3389/fmicb.2016. 01005

81. Duguma D, Hall MW, Smartt CT, Neufeld JD (2017) Temporal variations of microbiota associated with the immature stages of two florida culex mosquito vectors. Microb Ecol 74:979-989. https://doi.org/10.1007/s00248-017-0988-9
82. Favia G, Ricci I, Damiani C et al (2007) Bacteria of the genus Asaia stably associate with Anopheles stephensi, an Asian malarial mosquito vector. Proc Natl Acad Sci U S A 104:9047-9051. https://doi.org/10.1073/pnas.0610451104

83. Gimonneau G, Tchioffo MT, Abate L et al (2014) Composition of Anopheles coluzzii and Anopheles gambiae microbiota from larval to adult stages. Infect Genet Evol 28:715-724. https://doi. org/10.1016/j.meegid.2014.09.029

84. Saab SA, Dohna HZ, Nilsson LKJ et al (2020) The environment and species affect gut bacteria composition in laboratory cocultured Anopheles gambiae and Aedes albopictus mosquitoes. Sci Rep 10:1-13. https://doi.org/10.1038/s41598-020-60075-6

85. Merritt RW, Dadd RH, Walker ED (1992) Feeding behavior, natural food, and nutritional relationships of larval mosquitoes. Annu Rev Entomol 37:349-376. https://doi.org/10.1146/annur ev.ento.37.1.349

86. Coon KL, Vogel KJ, Brown MR, Strand MR (2014) Mosquitoes rely on their gut microbiota for development. Mol Ecol 23:27272739. https://doi.org/10.1111/mec. 12771

87. Kim CH, Lampman RL, Muturi EJ (2015) Bacterial communities and midgut microbiota associated with mosquito populations from Waste Tires in East-Central Illinois. J Med Entomol 52:63-75. https://doi.org/10.1093/jme/tju011

88. Moll RM, Romoser WS, Modrzakowski MC et al (2001) Meconial peritrophic membranes and the fate of midgut bacteria during mosquito (Diptera: Culicidae) metamorphosis. J Med Entomol 38:29-32. https://doi.org/10.1603/0022-2585-38.1.29

89. Romoser WS, Moll RM, Moncayo AC, Lerdthusnee K (2000) The occurrence and fate of the meconium and meconial peritrophic membranes in pupal and adult mosquitoes (Diptera: Culicidae). J Med Entomol 37:893-896. https://doi.org/10.1603/ 0022-2585-37.6.893

90. MacLeod HJ, Dimopoulos G, Short SM (2021) Larval diet abundance influences size and composition of the midgut microbiota of Aedes aegypti Mosquitoes. Front Microbiol 12:1-14. https:// doi.org/10.3389/fmicb.2021.645362

91. Wang Y, Gilbreath TM, Kukutla P et al (2011) Dynamic gut microbiome across life history of the malaria mosquito anopheles gambiae in Kenya. PLoS ONE 6:1-9. https://doi.org/10.1371/ journal.pone. 0024767

92. Ellegaard KM, Engel P (2016) Beyond 16S rRNA community profiling: intra-species diversity in the gut microbiota. Front Microbiol 7:1-16. https://doi.org/10.3389/fmicb.2016.01475

93. Paris L, Peghaire E, Moné A et al (2020) Honeybee gut microbiota dysbiosis in pesticide/parasite co-exposures is mainly induced by Nosema ceranae. J Invertebr Pathol 172:107348. https://doi.org/10.1016/j.jip.2020.107348

94. Björkroth J, Dicks LMT, Endo A (2014) The genus Weissella. In: Wood BJB (ed) Holzapfel WH. Lactic Acid Bacteria. John Wiley \& Sons Ltd, Chichester, pp 417-428

95. Fusco V, Quero GM, Cho G-S et al (2015) The genus Weissella: taxonomy, ecology and biotechnological potential. Front Microbiol 6:e155. https://doi.org/10.3389/fmicb.2015.00155

96. Abriouel H, Lerma LL, del Casado Muñoz M, C et al (2015) The controversial nature of the Weissella genus: technological and functional aspects versus whole genome analysis-based pathogenic potential for their application in food and health. Front Microbiol 6:1-14. https://doi.org/10.3389/fmicb.2015.01197

97. Praet J, Meeus I, Cnockaert M et al (2015) Novel lactic acid bacteria isolated from the bumble bee gut: Convivina intestini gen. nov., sp. nov., Lactobacillus bombicola sp. nov., and Weissella bombi sp. nov. Antonie van Leeuwenhoek 107:1337-1349. https://doi.org/10.1007/s10482-015-0429-z

98. Ballinger MJ, Perlman SJ (2019) The defensive Spiroplasma. Curr Opin Insect Sci 32:36-41. https://doi.org/10.1016/j.cois. 2018.10.004 
99. Binetruy F, Bailly X, Chevillon C et al (2019) Ticks and tickborne diseases phylogenetics of the Spiroplasma ixodetis endosymbiont reveals past transfers between ticks and other arthropods. Ticks Tick Borne Dis 10:575-584. https://doi.org/10. 1016/j.ttbdis.2019.02.001

100. Anbutsu H, Fukatsu T (2011) Spiroplasma as a model insect endosymbiont. Environ Microbiol Rep 3:144-153. https://doi. org/10.1111/j.1758-2229.2010.00240.x

101. Anbutsu H, Fukatsu T (2003) Population dynamics of malekilling and non-male-killing spiroplasmas in Drosophila melanogaster. Appl Environ Microbiol 69:1428-1434. https://doi.org/ 10.1111/j.1574-6941.2006.00087.x

102. Song Y-N, Zhang W-J, Bi S-F et al (2015) New ansamycin analogues from the mutant strain of Streptomyces seoulensis. J Antibiot (Tokyo) 68:757-759. https://doi.org/10.1038/ja.2015.65

103. Brandt GEL, Blagg BSJ (2011) Monoenomycin: a simplified trienomycin A analogue that manifests anticancer activity. ACS Med Chem Lett 2:735-740. https://doi.org/10.1021/ml200108y

104. Mosaei H, Molodtsov V, Kepplinger B et al (2018) Mode of action of Kanglemycin A, an ansamycin natural product that is active against rifampicin-resistant mycobacterium tuberculosis. Mol Cell 72:263-274.e5. https://doi.org/10.1016/j.molcel.2018. 08.028

105. Funayama S, Cordell GA (2000) Ansamycin antibioticsA discovery, classification, biosynthesis and biological activities. In:
Studies in Natural Products Chemistry, pp 51-106. https://doi. org/10.1016/S1572-5995(00)80127-1

106. Olademehin OP, Kim SJ, Shuford KL (2021) Molecular dynamics simulation of atomic interactions in the vancomycin binding site. ACS Omega 6:775-785. https://doi.org/10.1021/acsomega.0c05353

107. Levine DP (2006) Vancomycin: a history. Clin Infect Dis 42:S5S12. https://doi.org/10.1086/491709

108. Säde E, Björkroth J (2019) Introduction to the Genera Pediococcus, Leuconostoc, Weissella, and Carnobacterium. In: Vinderola G, Ouwehand AC, Salminen S, von Wright A (eds) Lactic Acid Bacteria, 5th Editio. CRC Press, pp 65-85

109. He Q, Luo J, Xu J-Z et al (2020) Morphology and transcriptome analysis of nosema bombycis sporoplasm and insights into the initial infection of microsporidia. mSphere 5:1-12. https://doi. org/10.1128/msphere.00958-19

110. Keeling PJ, Corradi N, Morrison HG et al (2010) The reduced genome of the parasitic microsporidian Enterocytozoon bieneusi lacks genes for core carbon metabolism. Genome Biol Evol 2:304-309. https://doi.org/10.1093/gbe/evq022

111. Katinka MD, Duprat S, Cornillott E et al (2001) Genome sequence and gene compaction of the eukaryote parasite Encephalitozoon cuniculi. Nature 414:450-453. https://doi.org/ $10.1038 / 35106579$ 